\title{
Vorläufige Mittheilungen
}

über

\section{das städtische Pockenhospital im IV. Bezirke zu Wien.}

Von

Dr. Heinrich Auspitz, Primararzt daselbst.

Die folgenden wenn auch kurzen Mittheilungen bedürfen bei der Ueberfülle statistischen Materials, welches die Blatternepidemien der letzten Jahre geliefert baben, einer Entschuldigung. Sie möge darin gefunden werden, dass das unter meiner Leitung stehende Hospital innerhalb des hier berührten Zeitraumes von 14 Monaten eine Krankenzahl von über 5000 Blatternkranken beherbergte, und dass über ein solches Material an Pockenkranken aus einem Hospitale meines Wissens bisher in keiner Epidemie statistische Angaben geliefert worden sind.

Das Blatternspital Wieden wurde am 1. Februar 1872 in dem der Commune angehörigen Hause Nr. 8 in der Karolyigasse eröffnet. Letzteres war bis zu dieser Zeit an das Wiedner allg. Spital vermiethet, zum grossen Theile zu Beamten-Wohnungen und Administrationslocalen verwendet und nur hie und da im Falle der Noth in einigen seiner Räumlichkeiten mit Kranken belegt worden. Es besteht aus 2 Stockwerken und einem gewölbten Erdgeschoss, welche drei Seiten eines Oblongums bilden und einen geräumigen mit Bäumen bepflanzten Hof einschliessen. Die Langseite des Hauses hat ihre Fenster nach Ost und West gerichtet, die beiden Schmalseiten nach Süd und Nord. In beiden Stockwerken findet sich je ein die Langseite fast ganz einnehmender Saal von $5^{\prime} 3^{\prime \prime}$ Breite, $16^{\prime} 2^{\prime \prime} 6^{\prime \prime}$ Länge und $1^{\prime} 5^{\prime \prime}$ Höhe im 1. Stocke; von $5^{\prime} 4^{\prime \prime}$ Breite, $20^{\prime} 6^{\prime \prime}$ und nur $1^{\prime} 4^{\prime \prime} 6^{\prime \prime \prime}$ Höhe im 2. Stocke. Die daran stossenden Räumlichkeiten in beiden Stockwerken sind grössere und kleinere Wohnzimmer, 
meist nur mit Fenstern an einer Seite versehen, die in keiner Weise die zur Belegung mit Kranken erforderlichen Eigenschaften an sich tragen. Von einer selbstständigen Ventilation ausser durch Oeffnen der Fenster war natürlich keine Rede. In vielen der Räume, z. B. im grossen Saale des 1. Stockwerkes herrschte noch durch Monate nach ihrer Belegung penetranter Jodgeruch von der früher darunter befindlichen Apotheke.

Die niedrigen, etwas dumpfen Parterre-Räume waren zum Theil durch die früher daselbst untergebrachte Spitals-Apotheke, Wäsche- und Badlocalitäten in einem sehr derouten Zustande und mussten bei Uebernahme des Spitales mit Ausnahme des sofort ausgerüsteten Bades und eines Waschraumes vorläufig unbenützt bleiben. Nur für das Aufnahms-Journal und die Administration konnten einige Zimmer nächst dem Eingang verwendet werden. Einige kleine ebenerdige Häuschen an einer Seite des Hofes wurden als Todtenkammer u. s. w. benützt.

Den 2. Februar übernahm ich mit einem Secundararzte, Herrn Dr. Gilek, das zu einem Spital erst zu adaptirende Gebäude und es wurde zunächst der grosse Saal des 2. Stockwerkes mit Kranken, welche das Wiedner allg. Spital dahin abgab, belegt. Die Einrichtung und Adaptirung der Räume erfolgte sodann schrittwêise mit der Zunahme der Epidemie, so dass erst gegen Ende Juli sämmtliche verfügbare Räume, auch jene des Erdgeschosses, mit Betten ausgerüstet waren und mit 250 Kranken (im Maximum des Belages) wirklich belegt wurden. 4-6 kleinere Wohnzimmer wurden als Extrazimmer für zahlende Kranke verwendet.

Es wurden von Seite des dirigirenden Arztes und seiner auf vier vermehrten Hilfsärzte sowie der Verwaltung die grössten Anstrengungen gemacht, um den ganz misslichen Verhältnissen entgegen zu arbeiten. Durch Niederreissung der Zwischenwände wurden kleinere Räume in grössere umgestaltet, die mangelnde Ventilation wurde durch die rigoroseste Ueberwachung der Fensterlüftung auch im Winter zu ersetzen gesucht. Die Zwischenräume zwischen den einzelnen (hölzernen) Betten wurden auf mindestens 3 Fuss festgehalten; das Einstellen der Betten grundsätzlich vermieden.

Solang es die Krankenzahl gestattete, wurde ein Zimmer nach dem andern von Zeit zu Zeit geräumt und durch einige Archiv fur Dermatologie und Syphilis, 1873, 2. 
Tage gelüftet. Traten bedeutendere Abscesse, Erisypele oder Gangrän auf, so wurden die Kranken sofort nach Möglichkeit isolirt. Im Hochsommer, als die Räume sämmtlich belegt waren, wurde in dem mit Bäumen und Gesträuch bepflanzten, mit Sitzbänken versehenen grossen Hofe ein längliches Zelt aus doppelter, auf Stangen gespannter und an den nach Nord und Süd gerichteten Seitenwänden durch Jalousien unterbrochener getheerter Leinwand aufgeschlagen, welches durch eine Zwischenwand in zwei Abtheilungen für Männer und Weiber getheilt wurde und 10-12 Betten enthielt. Die Kranken blieben in diesẽm Zelte - falls nicht heftiges Ungewitter eintrat, - mit Behagen auch während der Nacht und es scheint, dass der Verlauf der schweren metastatischen Processe, welche daselbst behandelt wurden, sich günstiger gestaltete, als dies im geschlossenen Raume der Fall gewesen wäre.

Als die Räume des Spitals im Laufe des Sommers für die immer mehr steigende Zunahme an Einlass begehrenden Blatternkranken nicht mehr auszureichen schienen, wurde über Beschluss des Gemeinderathes am 1. Juli eine ebenerdige aus 4 grossen Sälen bestehende Holzbarake mit circa 100 Betten auf der etwa $1 / 2$ Stunde entfernten Siebenbrunnerwiese eröffnet, in welche sämmtliche Reconvalescenten des Spitals beständig transferirt wurden. Dadurch gestaltete sich der Krankenbelag im Communalspitale in der Art, dass sämmtliche 200-250 Betten während der Monate Juli bis October (die Barake ward am 5. October geschlossen) mit fiebernden, in der Krankheits-Akme befindlichen Kranken belegt waren.

$\mathrm{Zu}$ den Uebelständen, welche nicht beseitigt werden konnten, zählte ferner der Mangel guten Trinkwassers im Hause selbst, dessen zwei Brunnen nach einer vorgenommenen chemischen Analyse des Wassers ausser Gebrauch für die Kranken gesetzt und nur für Waschwasser etc. weiter benutzt wurden. Es musste daher das Trinkwasser ausserhalb des Hauses aus einem von der Kaiser Ferdinands-Wasserleitung mit filtrirtem Donauwasser gespeisten Bassin bezogen werden.

Endlich machte das Fehlen einer Wasserleitung im Hause und in den Stockwerken es nöthig, dass die Reconvalescenten sich über den Hof in das Badegemach begeben mussten und 
nur für die Schwerkranken und für die hydrotherapentischen Actionen Wasser in die Stockwerke getragen werden konnte.

Die Retiraden münden sämmtlich auf die Gänge und sind von den Krankenzimmern nur durch einfache Mauern getrennt. $\mathrm{Da}$ sie nicht als Closets eingerichtet, sondern in primitiver Weise direct mit dem Unrathscanal verbunden sind, war ihre Reinhaltung und Desodorisation mit grossen Schwierigkeiten verbunden. Dieselbe geschah durch häufiges Eingiessen von mit Kupfervitriol versetztem Wasser, durch oftmalige Säuberung und durch das strenge Verbot, Abfälle in dieselben zu werfen. Für letztere, insbesondere für abgenutzte Verbandstoffe und für die abfallenden Variolaborken wurden zwei grosse mit Deckeln versehene Tonnen im Hofraume aufgestellt und deren regelmässige Abfuhr überwacht. Für die Desinfection der im Hause selbst gewaschenen Kranken- und Bettwäsche wurde durch Eintauchen derselben in Carbolsäure-Lösung gesorgt, die Kleider der Kranken vor ihrer Entlassung durch heisse Dämpfe gezogen.

So waren die Verhältnisse des Hauses, welches den grössten Theil der öffentlich verpflegten Variolakranken Wiens während der Epidemie des Jahres 1872-1873 aufnahm. Es unterliegt keinem Zweifel, dass sie von den für ein Spital mit Recht geforderten Einrichtungen in vielen Beziehungen ausserordentlich abwichen und dass manche Umstände, wie der völlige Mangel einer Ventilation und einer Wasserleitung, die geringe Höhe der grossen Säle und die auf die eine Seite beschränkte Lage der Fenster in den kleinen Krankenzimmern, endlich die ungünstige Beschaffenheit und Lage des Bades, der Aborte u. s. w., zusammengenommen ein sehr unglückliches Heilungspercent erwarten lassen mussten.

Zum Glück hat sich diese Furcht nicht bewährt, wie aus dem folgenden Ausweise der Krankenbewegung vom 1. Februar 1872 bis 31 . März 1873 , also in 14 Monaten hervorgeht.

Aufgenommen wurden 5009 Kranke, davon wurden:

Aus dem Stande gebracht 4924, nämlich

Geheilt entlassen . . . 4460

Es starben . . . . . 464, d. i. 9.4 pCt.

$\mathrm{Zu}$ Ende März 1873 verblieben 85

Summa 5009 Kranke. 
Die Mortalität war also eine im Verhältniss zu den bekannten Resultaten der Spitäler in anderen schweren Epidemien und zu jenen der anderen Wiener Blatterspitäler während dieser Epidemie äusserst gering.

Der Krankheitsverlauf gestaltete sich in interessanter Weise verschieden in den einzelnen Monaten und es scheint evident aus der Vergleichung der einzelnen Daten folgende Thatsache hervorzugehen: So lange die gewöhnlichen Belagsverhältnisse wie in jedem anderen Spitale herrschten, also von Februar bis Juli war die Anzahl der Nachkrankheiten und Metastasen eine auffallend geringe. Von der Zeit an, da das ganze Haus mit Schwerkranken belegt war, nahmen die Metastasen und insbesondere schwere Augenerkrankungen an Zahl constant zu und sind seitdem auch nach Herstellung des normalen Belegungsmodus im October nicht mehr herabgedrückt worden.

Was die statistischen Verhältnisse der Kranken weiter betrifft, so wurden weitaus die meisten im Alter von 11-30 Jahren aufgenommen. Kinder unter 10 Jahren kamen gar nicht zur Aufnahme. Vom 30. Jahre an sank die Zahl der Blatternkranken immer mehr und war vom 50.-70. Jahre die geringste.

\begin{tabular}{|c|r|r|r|}
\hline Alter & $\begin{array}{c}\text { Geheilt } \\
\text { entlassen }\end{array}$ & Gestorben & Zusammen \\
\hline Vom 11.-20. Jahre & 2112 & 114 & 2226 \\
$21 .-30$. & 1878 & 192 & 2070 \\
$31 .-40$. & 334 & 104 & 438 \\
$41 .-50$. & 99 & 34 & 133 \\
$51,-60$. & 30 & 13 & 43 \\
$61 .-70$. & 5 & 7 & 12 \\
$71 .-80$. & 2 & - & 2 \\
\hline
\end{tabular}

Die nun folgende Tabelle gibt Aufschluss über das Ver- 
hältniss der Impfung zu den Blattern, soweit es im Hospitale zur Beobachtung kam:

Von den 4924 aus dem Stande Gebrachten sind 51 nicht Blatternkranke abgerechnet.

\begin{tabular}{|c|c|c|c|c|}
\hline & Genesen & Gestorben & Zusammen & $\begin{array}{c}\text { Mortalitäts- } \\
\text { procent }\end{array}$ \\
\hline a) Nicht geimpft ... & 155 & 76 & 231 & $33 \mathrm{pCt}$ \\
\hline nicht eruirbar bei . & 111 & 52 & 163 & $32 \mathrm{pCt}$ \\
\hline $\begin{array}{l}\text { c) Angeblich geimpft } \\
\text { aber ohne sichtbare }\end{array}$ & & & & \\
\hline Narben Geimpft mit deut- & 183 & 84 & 267 & 31 pCt. \\
\hline lichen Narben . . . & 3888 & 242 & 4130 & $5.8 \mathrm{pCt}$ \\
\hline $\begin{array}{l}\text { e) Zum zweiten Mal } \\
\text { geblattert . . . }\end{array}$ & 30 & 7 & 37 & 19 pCt. \\
\hline $\begin{array}{l}\text { f) Zum dritten Mal } \\
\text { geblattert . . . }\end{array}$ & 1 & - & 1 & - \\
\hline g) Revaccinirt $\cdots$ & 41 & 3 & 44 & $6.8 \mathrm{pCt}$ \\
\hline Summe . . & 4409 & 464 & 4873 & \\
\hline
\end{tabular}

Rechnet man nun die Rubriken $b$ und $c$ zu den Ungeimpften.a, so ergibt 'sich statt 33 pCt. Mortalität der Ungeimpften blos $32 \mathrm{pCt}$.

Rechnet man dagegen $b$ und $c$ zu den Geimpften $d$, so ergibt sich statt 5.8 pCt. Mortalität der Geimpften ein Procent von 8.3. Somit schwankt die Mortalität

der Geimpften zwischen 5.8 und $8.3 p C t$. der Ungeimpften zwischen 33 und $32 p C t$., ein Verhältniss, welches über den Werth der Impfung keinen Zweifel übrig lässt.

Aus dem obigen Ausweise geht noch hervor, dass die Revaccination in den zum Hospital ihre Zuflucht nehmenden Volksschichten in Wien fast gar nicht vorkommt. 
Die mir vorliegenden Daten über die Provenienz der Kranken nach den Stadtbezirken, ferner über das Verhältniss der Geschlechter zu einander, über die Berufsarten der Kranken u. s. w. lasse ich vorläufig bei Seite.

Ueber die pathologischen Beobachtungen und therapeutischen Resultate, welche sich im Verlaufe der Epidemie ergeben haben, wird baldmöglichst Bericht erstattet werden. Ich erwähne hier nur, dass nebst zahlreichen anderen Versuchen die IIydrotheraphie, gestützt auf eine gewissenhafte Prüfung der Eigenwärme, in grossem Maasstabe Anwendung gefunden hat. 